\title{
Characterization of Interactions Among CYP1A2, CYP2B4, and NADPH-cytochrome P450 Reductase: Identification of Specific Protein Complexes ${ }^{\mathbb{}}$
}

\author{
J. Patrick Connick, James R. Reed, and Wayne L. Backes \\ Department of Pharmacology and Experimental Therapeutics and Stanley S. Scott Cancer Center, Louisiana State University Health \\ Sciences Center, New Orleans, Louisiana
}

Received September 14, 2017; accepted December 7, 2017

\begin{abstract}
Cytochromes P450s (P450s) catalyze oxygenation reactions via interactions with their redox partners. However, other proteins, particularly other P450s, also have been shown to form complexes that modulate P450 function. Previous studies showed that CYP1A2 and CYP2B4 form a complex when reconstituted into phospholipid vesicles; however, details of the interactions among the P450s and NADPH-cytochrome P450 reductase (POR) have not been fully characterized. The goal of this study was to examine P450 complex formation in living cells, using bioluminescence resonance energy transfer (BRET). Various pairs of P450 and POR constructs were tagged with either green fluorescent protein or Renilla luciferase, and transfected into human embryonic kidney 293T cells. Complexes were demonstrated by measuring energy transfer between the tags, and disruption of the complex was verified by cotransfection with
\end{abstract}

unlabeled P450-system proteins. CYP1A2 and CYP2B4 formed a stable complex that could not be disrupted by cotransfection of untagged POR. Interactions of both P450s with POR were detected, with untagged CYP1A2 disrupting the POR-CYP2B4 interaction. In contrast, untagged CYP2B4 did not affect the POR-CYP1A2 interaction. These data are consistent with POR preferentially binding to the CYP1A2 moiety of CYP1A2-CYP2B4. BRET-detectable homomeric CYP1A2-CYP1A2 also was detected, and was disrupted by cotransfection of either POR or CYP2B4. Both CYP1A2 and CYP2B4 activities were affected by their coexpression in a manner consistent with formation of the high-affinity POR-CYP1A2-CYP2B4 complex. These findings demonstrate that CYP1A2 and CYP2B4 form a heteromeric POR-CYP1A2-CYP2B4 complex in living cells that has altered catalytic activities relative to the homomeric enzymes.

\section{Introduction}

The cytochromes P450s (P450s) are a superfamily of hemoproteins responsible for the metabolism of a majority of prescribed drugs (Guengerich, 2006; Lynch and Price, 2007). In the endoplasmic reticulum (ER), $\mathrm{P} 450$ s require physical interaction with their redox partners NADPH-cytochrome $\mathrm{P} 450$ reductase (POR) and cytochrome $b_{5}$ to accept the electrons required for activity. P450s substantially outnumber POR in the ER of most tissues (Peterson et al., 1976; Reed et al., 2011), and the ER contains numerous P450s (Zanger and Schwab, 2013), each requiring physical interaction with POR to metabolize substrates. This raises questions regarding how $\mathrm{P} 450$ system proteins are organized to facilitate catalysis effectively. Early studies suggested that P450 enzymes would compete for available POR as predicted by mass action (West and Lu, 1972; Miwa et al., 1979); however, in many cases simple competition could not fully explain the observed effect of one

This work was supported, in part, by the National Institutes of Health National Institute of General Medical Sciences [Grant GM123253]; and the National Institutes of Health National institute of Environmental Health Sciences [Grants ES004344 and ES013648].

https://doi.org/10.1124/dmd.117.078642.

SThis article has supplemental material available at dmd.aspetjournals.org.
P450 on another (Kaminsky and Guengerich, 1985; Dutton et al., 1987; Cawley et al., 1995; Backes et al., 1998).

Direct evidence that one P450 affects the function of another P450 was shown using mixed reconstituted systems containing CYP1A2, CYP2B4, and subsaturating POR. Under these conditions, CYP2B4 activities were inhibited, whereas CYP1A2 activities were stimulated (Backes et al., 1998). These results could not be explained by simple competition between the P450s for POR, and led to the hypothesis that CYP1A2 and CYP2B4 formed a complex that caused the high-affinity binding of POR to the CYP1A2 moiety (Backes et al., 1998; Kelley et al., 2005, 2006). These functional changes required both proteins to be in the same vesicles; when the different P450s were reconstituted into separate vesicles neither inhibition of CYP2B4 nor activation of CYP1A2 activities was observed. Additionally, physical CYP2B4CYP1A2 complexes were detected in mixed reconstituted systems by crosslinking/immunoprecipitation (Reed et al., 2010) and in detergent solutions by Förster resonance energy transfer using forms of the enzymes that were labeled with fluorescent probes (Davydov et al., 2001). Evidence for interactions between these proteins also was observed in microsomal preparations (Cawley et al., 2001). Taken together, the data support the existence of a POR-CYP1A2-CYP2B4 ternary complex at subsaturating POR.

Interactions between P450s are not unique to the CYP1A2/CYP2B4 system. Heteromeric complexes have been reported either by demonstrating

ABBREVIATIONS: BRET, bioluminescence resonance energy transfer; ER, endoplasmic reticulum; EROD, ethoxyresorufin-O-deethylation; GFP, green fluorescent protein; HEK, human embryonic kidney; P450, cytochrome P450; PBS, phosphate-buffered saline; POR, NADPH-cytochrome P450 reductase; PROD, pentoxyresorufin-O-dealkylation; Rluc, Renilla luciferase. 
physical complexes between the P450s (e.g., human CYP2C9/CYP3A4 and rat CYP1A1/CYP3A2) (Alston et al., 1991; Subramanian et al., 2010) or by showing that their presence in mixed systems affects $P 450$ function (CYP1A2/CYP2E1, CYP2C9/CYP2D6, CYP3A4/CYP2E1, and CYP2C9/CYP3A4) (Kelley et al., 2006; Subramanian et al., 2009, 2010; Davydov et al., 2015). Homomeric complexes have also been shown with CYP2C8, CYP2E1, CYP1A2, and CYP3A4 (Jamakhandi et al., 2007; Fernando et al., 2008; Hu et al., 2010; Reed et al., 2012; Davydov et al., 2013, 2015).

The goal of this study was to demonstrate that POR, CYP1A2, and CYP2B4 exist in cellular systems as a stable ternary complex. This was accomplished by expressing these proteins in human embryonic kidney (HEK) 293T cells and detecting the presence of complexes using bioluminescence resonance energy transfer (BRET). The results show that CYP1A2 and CYP2B4 form a stable complex in membranes, and that POR preferentially binds to the CYP1A2 moiety of the CYP1A2CYP2B4 complex, resulting in $\mathrm{P} 450$-specific alterations in catalytic activities. Furthermore, CYP1A2 forms homomeric complexes that are disrupted by the presence of excess POR.

\section{Materials and Methods}

Materials. Dulbecco's modified Eagle's medium, phosphate-buffered saline (PBS), fetal bovine serum, and Lipofectamine 2000 were purchased from Invitrogen (Eugene, OR). The plasmids used to generate BRET vectors (pGFP ${ }^{2}-\mathrm{N} 1, \mathrm{pGFP}^{2}-\mathrm{N} 2$, pRluc-N2, and pRluc-N3) and the $\mathrm{pGFP}^{2}$-Rluc vector were obtained from BioSignal Packard (Waltham, WA). GFP ${ }^{2}$ is a wild-type green fluorescent protein (GFP) that has been modified by a F64L substitution mutation, which results in brighter fluorescence but similar excitation and emission spectra. HEK-293T/17 cells were obtained from ATCC (Manassas, VA). Antibiotic-antimycotic solution was purchased from Life Technologies (Carlsbad, CA). Coelenterazine 400A was purchased from Gold Biotechnology (St. Louis, MO), and coelenterazine $\mathrm{h}$ was purchased from Promega (Madison, WI). 7-Pentoxyresorufin and 7-ethoxyresorufin were purchased from Anaspec (Fremont, CA), and cytochrome $c$ was obtained from Sigma (St. Louis, MO).

Generation of BRET Vectors. All of the P450s and POR were wild-type proteins without any modifications to their amino acid sequences. To generate the BRET expression vectors, linear cDNA coding for full-length, wild-type rabbit CYP1A2 (NM_001171121), CYP2B4 (NM_001170859), and POR (NM_001160290) was amplified from existing bacterial expression vectors with polymerase chain reaction using primers to introduce restriction sites that immediately flanked the full length gene, excluding the stop codon. The restriction sites (with the $5^{\prime}$ site listed first) were: EcoRI and BamHI for CYP1A2, NheI and EcoRI for CYP2B4, and EcoRI and HindIII for POR. These polymerase chain reaction products were then ligated into the empty vectors ( $\mathrm{pGFP}^{2}-\mathrm{N} 1$ and pRluc-N2 for the P450s, pGFP ${ }^{2}-\mathrm{N} 3$ and pRluc-N1 for POR) after each was digested by the indicated pair of restriction enzymes (New England Biolabs Inc., Ipswich, MA). The multiple cloning sites of the vectors are upstream of the GFP or Renilla luciferase (Rluc) tag. This information is summarized in Supplemental Table 1. This orientation was chosen to ensure that the heterologous protein tags were less likely to interfere with the ability of the proteins' $\mathrm{N}$-terminal membranebinding regions to insert into the ER membrane. Due to the restriction sites used, the BRET vectors code for a 5-20 amino acid sequence between the 3 ' end of the inserted gene and the start codon for the GFP or Rluc tag. To generate vectors for the expression of untagged, wild-type CYP1A2, CYP2B4, and POR, site-directed mutagenesis was performed to create a stop codon immediately $3^{\prime}$ to the main protein sequence. Site-directed mutagenesis was performed using the QuikChange II kit from Agilent Technologies (Santa Clara, CA). All polymerase chain reaction amplification and mutagenesis primers were purchased from Integrated DNA Technologies (Coralville, IA). Insertion of cDNA and mutagenesis were confirmed by sequencing (ACGT, Germantown, MD).

Cell Culture and Transfection. HEK-293T/17 cells were maintained under a humidified $37^{\circ} \mathrm{C}$ atmosphere supplemented with $5 \% \mathrm{CO}_{2}$. Cell growth media consisted of Dulbecco's modified Eagle's medium supplemented with $10 \%$ fetal bovine serum and $1 \times$ antibiotic-antimycotic solution $(100 \mathrm{U} / \mathrm{ml}$ penicillin, $100 \mu \mathrm{g} / \mathrm{ml}$ streptomycin, and $250 \mathrm{ng} / \mathrm{ml}$ amphotericin B). Six-well plates were seeded with cells such that they were $>90 \%$ confluent at the time of transfection the following day. Transfections were performed using preformed complexes of Lipofectamine 2000 with 1 to $2 \mu \mathrm{g}$ DNA per well. Empty plasmid (pUC19) was used to ensure all transfections contained the same quantity of DNA.

BRET Assays. For BRET assays, cells were first transfected with $1 \mu \mathrm{g}$ total DNA at different ratios of the GFP and Rluc constructs. The goal for these transfections was to generate a series of cells expressing the same total protein at a range of GFP:Rluc ratios to ensure that the BRET complexes were specific and not due to changes in protein concentration. If this transfection strategy yielded approximately the same total protein expression across all conditions, the results were considered valid. In most cases, however, one or two additional trials were necessary to achieve constant levels of protein expression. After allowing at least 24 hours for protein expression, cells were checked using fluorescence microscopy to ensure efficient expression of the GFP fusion proteins. Each transfection was performed with the following three controls: a GFP-Rluc fusion protein, the Rluc fusion protein alone, and cells transfected with only pUC19. Cells were harvested in $1 \mathrm{ml}$ PBS, centrifuged, and resuspended in $700 \mu \mathrm{l}$ PBS. For BRET measurements, $100 \mu \mathrm{l}$ of suspended cells were distributed in quadruplicate into an opaque white 96-well plate. A TriStar LB 941 microplate reader (Berthold Technologies, Bad Wildbad, Germany) was used for BRET measurements. This plate reader was programmed to perform the following actions for each well: inject $100 \mu \mathrm{l}$ of a $10 \mu \mathrm{M}$ coelenterazine 400A/PBS solution, shake for 1 second to mix, read Rluc emission for 3 seconds at $410 \mathrm{~nm}$, and read GFP emission for 3 seconds at $515 \mathrm{~nm}$. To calculate the BRET ratio, the average GFP and Rluc emission values from the untransfected control cells were used to zero each individual measurement, and then the raw BRET signal was calculated by dividing the GFP signal by the Rluc signal. Finally, the net BRET value was obtained by subtracting the baseline BRET ratio generated by cells expressing Rluc-tagged protein alone.

Determination of Relative Protein Expression. Relative expression levels of GFP- and Rluc-tagged proteins were determined photometrically by comparison with cells expressing a GFP-Rluc fusion protein. First, GFP expression was determined by measuring fluorescence ( $410 \mathrm{~nm}$ excitation; $515 \mathrm{~nm}$ emission) on a SpectraMax M5 plate reader using $100 \mu \mathrm{l}$ samples of the original cell suspension in black clear-bottomed 96-well plates (Corning Inc., Corning, NY). Rluc expression was measured in the microplate reader using the fourth quadruplicate of each experimental condition. Coelenterazine $\mathrm{h}$ was added to these wells to a final concentration of $5 \mu \mathrm{M}$, and unfiltered emission was measured for 1 second. The GFP and Rluc signals of each sample were then normalized to that of the GFP-Rluc fusion protein (which is assumed to have a 1:1 GFP:Rluc expression ratio), such that dividing the normalized GFP value by the normalized Rluc value yielded an approximation of the actual GFP:Rluc expression ratio.

Activity Measurements in HEK-293T/17 Cells. Three groups of cells were prepared by transfecting with vectors coding for CYP1A2-GFP, CYP2B4-GFP, or both. After 36 hours, cells were harvested, incubated on ice in hypotonic media (10 mM HEPES, $1.5 \mathrm{mM} \mathrm{MgCl}, 10 \mathrm{mM} \mathrm{KCl}, 0.5 \mathrm{mM}$ dithiothreitol), and lysed by passing through a 27 -gauge needle 10 times. The lysate was centrifuged at $10,000 \mathrm{~g}$ for 20 minutes to pellet debris. The supernatant was collected and centrifuged at $110,000 \mathrm{~g}$ for 1 hour after which the pellet was resuspended in $500 \mu \mathrm{l}$. GFP fluorescence was measured in 1:20 dilutions of each sample to estimate the GFP-tagged P450 concentration relative to a previously generated standard curve using western blot densitometry. Four reaction conditions were then prepared based on these data: microsomes containing CYP1A2-GFP alone and CYP2B4-GFP alone, and microsomes containing both CYP1A2-GFP and CYP2B4-GFP. Samples were arranged in quadruplicate in a 96-well clear-bottom plate with added substrate (7-ethoxyresorufin or 7-pentoxyresorufin) and incubated for 5 minutes at $37^{\circ} \mathrm{C}$ in a SpectraMax M5 plate reader. The rates of ethoxyresorufin- $O$-dealkylation (EROD) and pentoxyresorufin- $O$-dealkylation (PROD) activity were then measured in real time by fluorescence after the reactions were initiated by addition of NADPH. To determine the relative expression of CYP1A2-GFP and CYP2B4-GFP in the cotransfected cells, samples were analyzed using SDS-polyacrylamide gel electrophoresis and western blotting with a GFP-specific antibody along with a purified GFP standard (Vector Laboratories, Burlingame, CA). POR levels were determined by measuring NADPH-dependent reduction of cytochrome $c$ (Sigma). Microsomes and cytochrome $c$ (final concentration $360 \mu \mathrm{M}$ ) in $0.1 \mathrm{mM}$ potassium phosphate buffer ( $\mathrm{pH} 7.25$ ) were incubated in quadruplicate in black, clear-bottom, 96-well plates at $37^{\circ} \mathrm{C}$ for 5 minutes. Reactions were initiated by the addition of NADPH 
and reaction progress was monitored by measuring the difference in absorbance between the 550 and the $541 \mathrm{~nm}$ isosbestic point as described previously (Yasukochi and Masters, 1976; Cawley et al., 2001). Known amounts of purified POR (Marohnic et al., 2011) were used to create a standard curve for quantification.

Using the measured protein concentrations, and previously determined $K_{\mathrm{D}}$ values between POR and CYP1A2 (Backes et al., 1998; Reed et al., 2012) and POR and CYP2B4 (Backes et al., 1998; Brignac-Huber et al., 2013), the expected activities for PROD and EROD were calculated under conditions where the cotransfected systems followed a model allowing for only simple competition using Dynafit 4 (Backes et al., 1998). The text files used can be found in Supplemental Fig. 1.

\section{Results}

Characterization of the Physical Complexes among CYP1A2, CYP2B4, and POR. In previous reports, we provided both kinetic and physical evidence that CYP1A2 and CYP2B4 form a heteromeric complex in reconstituted systems (Backes et al., 1998; Reed et al., 2010) and microsomes (Cawley et al., 2001). Based on kinetic data, formation of this heteromeric complex causes the selective high-affinity association of POR to the CYP1A2 moiety of this complex. The goal of this study was to demonstrate that the POR-CYP1A2-CYP2B4 complex is formed in the ER of living cells after recombinant expression, and more specifically, to identify the specific physical protein-protein interactions involved. This was accomplished by tagging CYP1A2, CYP2B4, and POR with either Rluc or GFP, after transient transfection into HEK-293T/17 cells. Complex formation was then measured using BRET.

The first step was to show that BRET can be used to detect CYP1A2: CYP2B4 complexes in HEK-293T/17 cells. This was done by transfecting CYP1A2-GFP and CYP2B4-Rluc constructs at various ratios such that total protein expression remained approximately constant. Under these conditions, a specific protein-protein interaction between the GFP- and Rluc-labeled proteins will yield a saturation curve as the relative level of GFP expression increases (James et al., 2006). The asymptote to which these curves trend at high GFP:Rluc (BRET max $_{\text {ax }}$ ) represents the condition in which every Rluc-tagged protein is maximally surrounded by a GFP-tagged protein. Changes in BRET $\max$ can, therefore, be interpreted as changes in the fraction of interacting BRET pairs. Nonspecific interactions generate a curve that is independent of the expression ratio. Figure 1A shows that we are able to detect an interaction between CYP1A2 and CYP2B4 using BRET.

If the CYP1A2-CYP2B4 complex is responsible for the previously observed changes in P450 activity (Backes et al., 1998), it should be refractory to disruption by POR. To determine if the CYP1A2-CYP2B4 complex was stable, HEK-293T/17 cells were transfected with CYP1A2-GFP and CYP2B4-Rluc both in the presence and absence of cotransfected unlabeled POR. The results (Fig. 1B) show that POR does not interfere with the BRET complex, and is consistent with CYP1A2CYP2B4 being stable in the presence of POR.

Although these data indicate that the CYP1A2-CYP2B4 complex is stable, they do not conclusively show that POR interacts with the P450s in the CYP1A2-CYP2B4 complex; nor do they show whether there is selective binding of POR to a particular moiety of that complex. Our previous kinetic data support the formation of a CYP1A2-CYP2B4 complex with POR selectively associating with the CYP1A2 moiety (Backes et al., 1998). If this is the case, a CYP2B4/POR BRET pair should generate a signal that is disrupted by the addition of unlabeled CYP1A2; however, BRET measured from a CYP1A2/POR BRET pair should not be affected by the addition of unlabeled CYP2B4. In the first experiment (Fig. 2), a BRET signal was measured in cells transfected with the CYP2B4-GFP/POR-Rluc BRET pair, clearly showing the
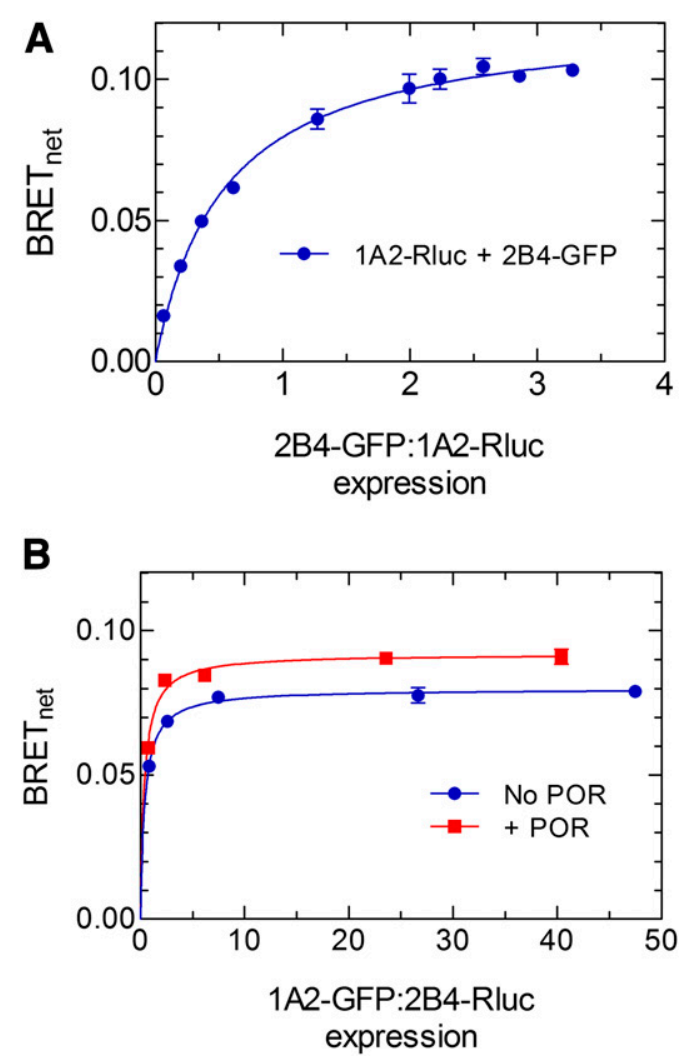

Fig. 1. Detection of physical complexes between CYP1A2 and CYP2B4 using BRET. (A) Physical complex formation between CYP2B4 and CYP1A2. pGFP2 N1/CYP2B4-GFP and pRluc-N2/CYP1A2-Rluc were transfected into HEK-293T/ 17 cells at different ratios and BRET was measured. (B) Effect of POR on the CYP1A2/CYP2B4 BRET pair. pGFP ${ }^{2}$-N1/CYP1A2-GFP and pRluc-N2/CYP2B4Rluc were cotransfected into HEK-293T/17 cells (blue). This was repeated in the presence of cotransfected unlabeled POR, which did not measurably affect BRET max $_{\text {ax }}$ (red), indicating that the CYP1A2-CYP2B4 complex is stable in the presence of POR. The mean \pm S.D. value for triplicate determinations is represented unless the error bars are smaller than the plotted points.

formation of a specific CYP2B4-POR complex. Cotransfection of unlabeled CYP1A2 caused a sharp decrease in BRET max $_{\text {for }}$ fhis pair. These data demonstrate that CYP1A2 disrupts the CYP2B4-POR interaction, and provides an explanation for the inhibition of CYP2B4 activity in the presence of CYP1A2 (Backes et al., 1998; Reed et al., 2010).

In the converse experiment, the effect of unlabeled CYP2B4 on the interaction between POR-Rluc and CYP1A2-GFP was examined. If POR selectively binds to the CYP1A2 moiety of a POR-CYP1A2CYP2B4 complex, the addition of unlabeled CYP2B4 would not be expected to disrupt POR-CYP1A2 binding. POR-Rluc and CYP1A2GFP were transfected into HEK-293T/17 cells, producing a BRET signal consistent with complex formation between the proteins (Fig. 3). Cotransfection of unlabeled CYP2B4 did not disrupt this complex. Taken together, these results are consistent with the presence of a PORCYP1A2-CYP2B4 trimer, where POR selectively binds to the CYP1A2 moiety of this complex.

Effect of Heteromeric Complex Formation on Monooxygenase Function. To solidify the link between these physical interactions and the previously seen functional effects, we measured the activity of the P450s in transfected cells. A factor that complicates analysis of these experiments was the difficulty in precisely controlling protein concentration in transfected cells, where cotransfection of one protein influences the expression of other transfected P450 system proteins. 


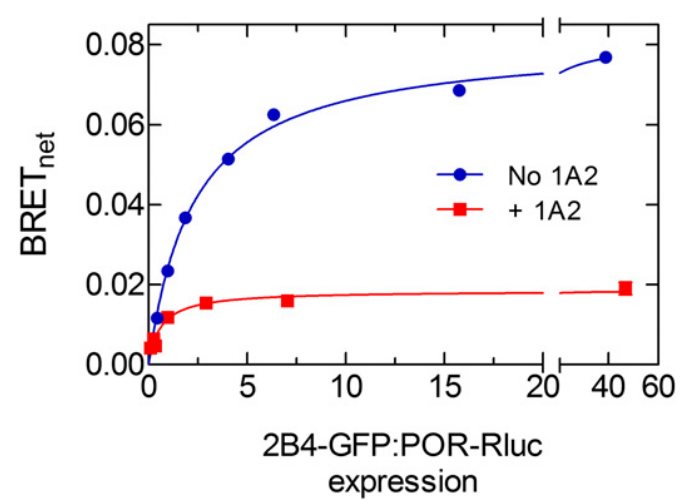

Fig. 2. Effect of unlabeled CYP1A2 on the POR/CYP2B4 BRET pair. pGFP ${ }^{2}-\mathrm{N} 1 /$ CYP2B4-GFP and pRluc-N1/POR-Rluc were cotransfected into HEK-293T/17 cells at varying GFP:Rluc ratios both in the absence (blue) and presence (red) of cotransfected, unlabeled CYP1A2. Each point represents the mean of triplicate determinations with the S.D. value within the data point.

Generally, cotransfection of a second or third P450 system protein leads to lower expression levels of the others. To circumvent this variability, the experimentally obtained activities were compared with those expected if CYP1A2 and CYP2B4 did not form a complex and simply competed for the available POR. CYP1A2-mediated EROD and CYP2B4-mediated PROD activities were measured using microsomes from cells that were transfected with CYP1A2-GFP, CYP2B4-GFP, or both. When both P450s were cotransfected, CYP2B4-mediated PROD activity was lower (Fig. 4A), whereas CYP1A2-mediated EROD activity was greater than that predicted by simple competition between the P450s for limiting POR (Fig. 4B). These data show that the presence of CYP2B4 enhances CYP1A2-mediated EROD activity, and the presence of CYP1A2 inhibits CYP2B4-mediated PROD activity, in a manner consistent with the expected characteristics of the CYP1A2CYP2B4 complex as determined from the studies with purified, reconstituted systems.

Characterization of Homomeric P450 Complexes in Cellular Systems. We previously demonstrated that CYP1A2 exhibits nonMichaelis-Menten, sigmoidal behavior when P450-dependent activities were measured as a function of POR concentration. Furthermore, it was shown that the ability of CYP1A2 to form homomeric complexes was retained in both reconstituted systems and the ER membrane of living cells. Formation of this complex was verified by the disruption of the CYP1A2-Rluc/CYP1A2-GFP BRET pair by the cotransfection of unlabeled CYP1A2 (Reed et al., 2012). These results suggested that the CYP1A2-CYP1A2 complex might be related to the sigmoidal kinetic response, and suggested that POR might be capable of disrupting the CYP1A2-CYP1A2 complex. To test this possibility, HEK-293T/17 cells were transfected with CYP1A2-GFP and CYP1A2-Rluc, and BRET measurements yielded the expected saturation curve (Fig. 5, blue). When this BRET pair was cotransfected with unlabeled POR, a curve with a lower BRET $_{\max }$ was generated, indicating that POR (at approximately 1:2 POR:CYP1A2 transfection ratio) decreased the proportion of multimeric CYP1A2 (Fig. 5, red). These data are consistent with the hypothesis that CYP1A2 can exist in the membrane, at least in part, as a homomeric complex that can be destabilized by the presence of POR.

Figure 6 shows the potential for homomeric complex formation of CYP2B4. Although the curve appears to exhibit saturation, the saturation does not occur at a 1:1 GFP:Rluc ratio as required for specific homomeric complexes (Mercier et al., 2002). Furthermore, the CYP2B4-GFP/CYP2B4-Rluc signal is not affected by cotransfection of unlabeled CYP2B4, suggesting either that CYP2B4 does not form a

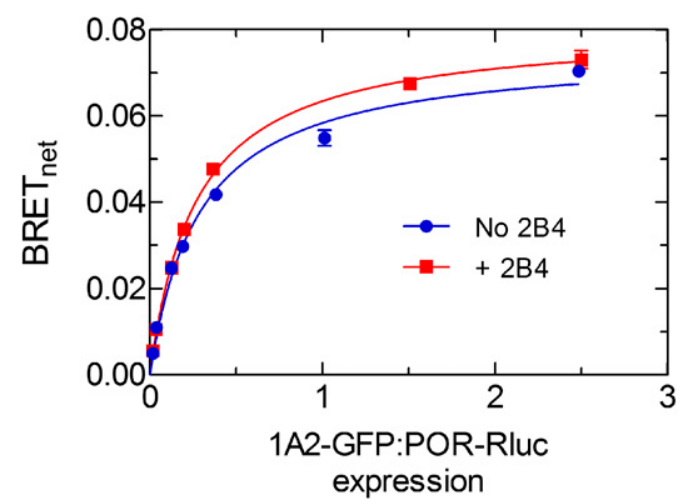

Fig. 3. Effect of unlabeled CYP2B4 on the BRET-sensitive POR-CYP1A2 complex. pGFP ${ }^{2}$-N1/CYP1A2-GFP and pRluc-N1/POR-Rluc were cotransfected into HEK-293T/17 cells at varying GFP:Rluc ratios in the absence (blue) and presence (red) of cotransfected unlabeled CYP2B4. Points represent the mean \pm S.D. values for three measurements; for most points, the error is smaller than the data point.

specific homomeric complex or that such a complex is not detectible due to the stringent conditions required to activate the GFP ${ }^{2} /$ Rluc BRET pair.

\section{Discussion}

Interactions between P450s not only have been established for several $\mathrm{P} 450 \mathrm{~s}$, but in many instances also have been shown to influence monooxygenase function (Hazai and Kupfer, 2005; Kelley et al., 2006; Subramanian et al., 2009, 2010; Reed et al., 2010; Davydov et al., 2015). The interaction between CYP1A2 and CYP2B4 is the most studied of these pairs, with kinetic experiments precluding the possibility that the two P450s were competing for POR by a simple mass-action process. In these studies, the apparent affinity of CYP1A2 for POR increased significantly in the presence of CYP2B4 (Backes et al., 1998). Similar functional effects were seen in microsomes purified from rabbit liver (Cawley et al., 2001), leading to the hypothesis that CYP1A2 and CYP2B4 exist in a heteromeric complex that affects P450 function. Davydov et al. (2001) subsequently published data showing that purified CYP1A2 and CYP2B4, in the presence of detergent Emulgen-913, formed heteromeric complexes using Förster resonance energy transfer. This work also corroborated the finding that POR preferentially bound to CYP1A2 when in the presence of CYP2B4 and substrate 7-ER (Davydov et al., 2001). Further work showed that the kinetic behavior of the binary P450 system, containing both CYP1A2 and CYP2B4, reverted to simple competition when the ionic strength of the solution was increased, which is consistent with the disruption of a P450 complex that is stabilized by electrostatic interactions (Kelley et al., 2005). The functional effects were then shown to occur only when CYP1A2 and CYP2B4 were reconstituted in the same lipid vesicles, and the formation of a physical complex between CYP1A2 and CYP2B4 was shown by chemical cross-linking/immunoprecipitation (Reed et al., 2010).

The work presented here more specifically focuses on the physical complexes that exist among POR, CYP1A2, and CYP2B4 in living cells. The results provide strong evidence for the presence of the CYP1A2-CYP2B4 complex in a living system whose interaction with POR strongly favors binding via the CYP1A2 moiety. In the simplest protein complex that describes the results, the dominant active species is a trimer with both POR and CYP2B4 bound to CYP1A2, but not to each other. The studies also showed that the characteristic functional effects of CYP1A2/CYP2B4 systems were retained in living cells, which both solidifies the role of physical complexes in these effects and shows that they exist in natural membranes. 


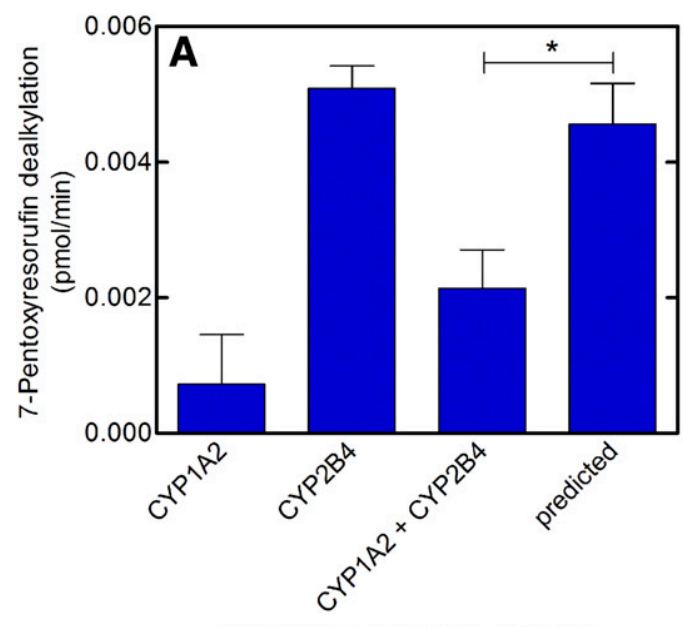

HEK293T cells transfected with:

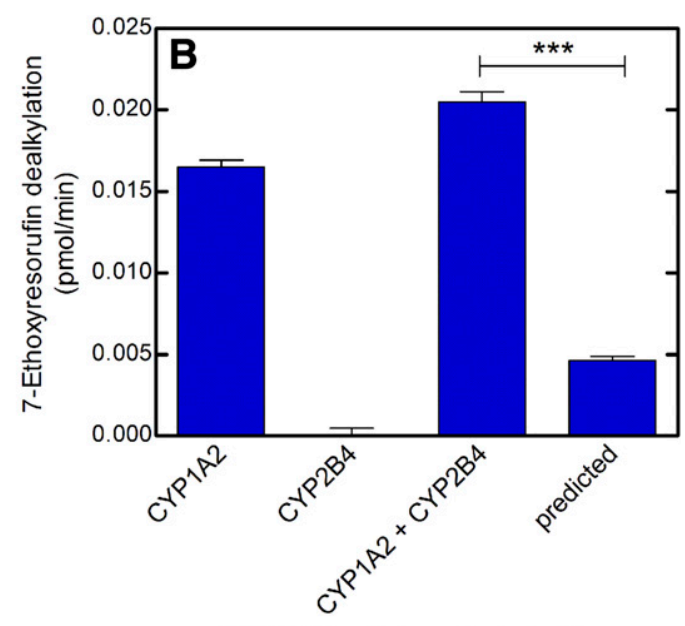

HEK293T cells transfected with:

Fig. 4. Effect of cotransfection of CYP1A2-GFP and CYP2B4-GFP on PROD and EROD in microsomes isolated from transfected HEK-293T/17 cells. Cells were transfected with pGFP ${ }^{2}-\mathrm{N} 1 / \mathrm{CYP} 1 \mathrm{~A} 2-\mathrm{GFP}, \mathrm{pGFP}^{2}-\mathrm{N} 1 / \mathrm{CYP} 2 \mathrm{~B} 4-\mathrm{GFP}$, or both. Microsomes derived from these cells were then incubated in the presence of either 7-pentoxyresorufin or 7-ethoxyresorufin, and the initial rate of resorufin accumulation was determined spectrofluorometrically. The experimentally determined rates were then compared with those predicted for CYP1A2 and CYP2B4 if they behaved as monomers that simply competed for the available POR. Because each group of transfected cells contained different levels of POR, CYP1A2, and CYP2B4, the predicted rates were determined using Dynafit 4, based on the model described in Supplemental Fig. 1. Consequently, if the P450 enzymes affected each other's function, the experimental data would be expected to deviate from the "predicted" activities. This analysis showed that cotransfection of both P450s led to lower rates of PROD activity (A) and higher rates of EROD activity (B) than predicted by simple competition. Protein concentrations from the CYP1A2 group were 0.048 and $0.005 \mu \mathrm{M}$ for CYP1A2 and POR, respectively. Concentrations for the CYP2B4 group were 0.040 and $0.005 \mu \mathrm{M}$ for CYP2B4 and POR, respectively. Concentrations for the group cotransfected with both CYP1A2 and CYP2B4 were 0.051, 0.045, and 0.005 $\mu \mathrm{M}$ for CYP1A2, CYP2B4, and POR, respectively. The activities represent the mean \pm S.E.M. values for four replicates. Unpaired $t$ test: $* P<0.05 ; * * P<0.001$ for the difference between the measured and predicted values.

In addition to its heteromeric interactions with POR and CYP2B4, CYP1A2 was shown to form homomeric complexes in a number of systems (Reed et al., 2012). Using kinetic analysis, our laboratory previously demonstrated that some, but not all, CYP1A2-dependent activities produce a sigmoidal response as a function of POR concentration, which becomes more prominent as the CYP1A2 concentration is increased. This response is consistent with the formation of a homomeric CYP1A2-CYP1A2 complex. Interestingly, the sigmoidal response can be converted to hyperbolic Michaelis-Menten behavior by increasing the ionic strength of the buffer-again consistent with the formation of a complex that is stabilized by ionic interactions. These kinetic data were corroborated by demonstration that CYP1A2 forms BRET-detectable physical homomeric complexes (Reed et al., 2012). Here, we extend this work and show that these BRET-detectable physical CYP1A2 complexes can be disrupted by the cotransfection of POR. This disruption corroborates earlier work showing that reconstituted CYP1A2 was $20 \%$ immobile alone and $11 \%$ immobile in the presence of POR when examined using rotational diffusion (Yamada et al., 1995). The evidence as a whole points to a system where CYP1A2 forms homomeric multimers that are either unable to associate with POR (Reed and Backes, 2017), or have a lower specific activity than monomeric CYP1A2. POR appears to be able to induce disruption of these complexes (as was seen in our previous work as reaction rates became proportional to CYP1A2 levels with excess POR). CYP2B4 appears to have a similar effect on the CYP1A2-CYP1A2 complex. Since CYP1A2 is a highly inducible P450, this effective deactivation at higher expression levels could act as a mechanism to balance the protein's oxygenase activity and uncoupled production of reactive oxygen species, an effect similar to that proposed in a recently presented model (Davydov, 2011; Davydov et al., 2015). As noted previously, with some substrates, the presence of CYP2B4 actually appears to have increased the activity of CYP1A2. This effect was seen again in microsomes prepared from HEK-293T cells transfected with both P450 enzymes (the same system as the BRET experiments).
Although BRET can definitively show whether two proteins form a complex, it is somewhat limited in that it cannot directly determine the affinities of protein-protein interaction or the size of the supramolecular complexes. The model of a CYP1A2-CYP2B4 dimer interacting as a unit with POR is the simplest explanation for our observations but we cannot rule out larger and/or more complex physical organization of these proteins. Additionally, because energy transfer depends on the orientation of the two chromophores in addition to their proximity, the lack of a strong BRET signal does not absolutely imply the lack of complex formation.

The $R_{0}$ value (i.e., the distance at which energy transfer efficiency is $50 \%$ of the maximum between a BRET pair) for Rluc and GFP was

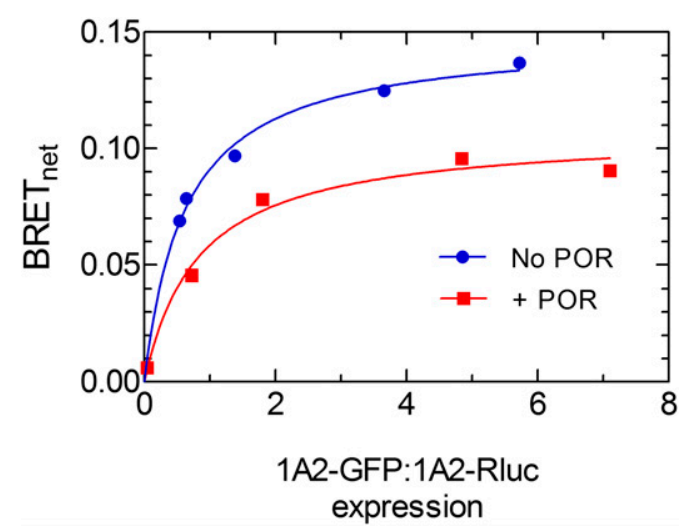

Fig. 5. Effect of unlabeled POR on the BRET signal for the CYP1A2-CYP1A2 complex. pGFP ${ }^{2}$-N1/CYP1A2-GFP and pRluc-N2/CYP1A2-Rluc were cotransfected into HEK-293T/17 cells, and BRET was measured (blue curve). This was repeated in the presence of cotransfected unlabeled POR (red curve). The decrease in BRET $_{\text {max }}$ is consistent with disruption of the CYP1A2-CYP1A2 complex. Each point represents the mean value of triplicate determinations with the S.D. value within the data points. 


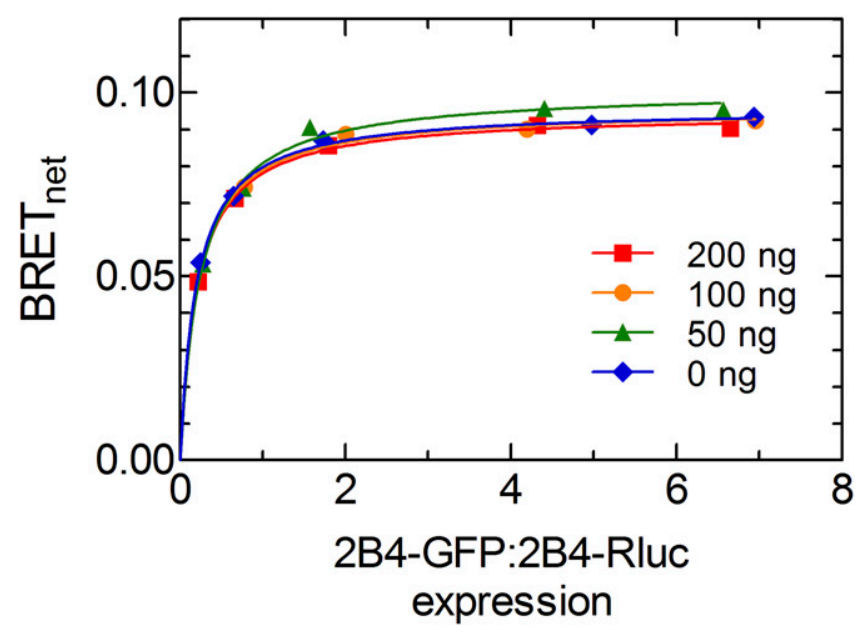

Fig. 6. Effect of unlabeled CYP2B4 on the BRET signal for the CYP2B4-CYP2B4 BRET pair. pGFP ${ }^{2}-\mathrm{N} 1 / \mathrm{CYP} 2 \mathrm{~B} 4-\mathrm{GFP}$ and pRluc-N2/CYP2B4-Rluc were cotransfected into HEK-293T/17 cells, and BRET was measured in the presence and absence of different amounts of cotransfected unlabeled CYP2B4. The presence of unlabeled competitor did not have an effect on the $\mathrm{BRET}_{\max }$, suggesting that CYP2B4-GFP and CYP2B4-Rluc do not form a specific BRET-detectible complex. Each point represents the mean value of triplicate determinations with the S.D. value within the data points.

experimentally determined to be $7.50 \pm 0.03 \mathrm{~nm}$ (Dacres et al., 2010). This distance is similar in scale to that of CYP1A2 as determined by its crystal structure (Sansen et al., 2007). Since the protein tags used in this work are appended to the C-terminus of each enzyme, and each enzyme has its orientation restricted by the $\mathrm{N}$-terminal membrane-binding domain, any proteins in physical contact would probably have their BRET pair within the $7.5 \mathrm{~nm}$ range. Proteins not in contact would quickly fall out of the range of efficient energy transfer.

GFP-tagged proteins were used as a means to confirm that tagged proteins retained activity, to facilitate the measurement of protein concentration at the time of the experiment, and to allow the direct comparison of both CYP1A2-GFP and CYP2B4-GFP on the same western blot by using a GFP antibody. Transfection with equal amounts of cDNA for each tagged P450 led to more expression of CYP2B4-GFP than CYP1A2-GFP. CYP1A2-GFP constituted 53\% of total GFPtagged protein expression in the final iteration of the experiment after adjusting the ratio of transfected DNA.

In summary, these experiments demonstrated that CYP1A2 and CYP2B4 form a stable heteromeric complex in living cells. Although POR is capable of a BRET-detectable complex with both CYP1A2 and CYP2B4, when both P450s are present in HEK-293T cells, POR binds selectively to the CYP1A2 moiety of the CYP1A2-CYP2B4 complex, which is consistent with the formation of POR-CYP1A2-CYP2B4 as the base complex. Formation of this complex has functional consequences, i.e., the stimulation of CYP1A2-mediated activities and the inhibition of CYP2B4-mediated activities. These effects, which have been well established with other in vitro systems, are also observed in HEK cells transfected with these proteins. Finally, CYP1A2 was shown to form a homomeric complex that could be disrupted by increasing POR concentrations. Taken together, these results suggest an intricate interplay between the relative concentrations of different P450 system proteins, their ability to form complexes, and their effect on $\mathrm{P} 450$ function. Future studies are required not only to identify the size of these supramolecular complexes, but also to determine how complex formation is affected by their localization into specific membrane regions (Brignac-Huber et al., 2011, 2016; Park et al., 2014, 2015).

\section{Authorship Contributions}

Participated in research design: Connick, Reed, Backes.

Conducted experiments: Connick, Reed.

Performed data analysis: Connick, Reed, Backes.

Wrote or contributed to the writing of the manuscript: Connick, Reed, Backes.

\section{References}

Alston K, Robinson RC, Park SS, Gelboin HV, and Friedman FK (1991) Interactions among cytochromes P-450 in the endoplasmic reticulum. Detection of chemically cross-linked complexes with monoclonal antibodies. J Biol Chem 266:735-739.

Backes WL, Batie CJ, and Cawley GF (1998) Interactions among P450 enzymes when combined in reconstituted systems: formation of a 2B4-1A2 complex with a high affinity for NADPHcytochrome P450 reductase. Biochemistry 37:12852-12859.

Brignac-Huber L, Reed JR, and Backes WL (2011) Organization of NADPH-cytochrome P450 reductase and CYP1A2 in the endoplasmic reticulum - microdomain localization affects monooxygenase function. Mol Pharmacol 79:549-557.

Brignac-Huber LM, Park JW, Reed JR, and Backes WL (2016) Cytochrome P450 organization and function are modulated by endoplasmic reticulum phospholipid heterogeneity. Drug Metab Dispos 44:1859-1866

Brignac-Huber LM, Reed JR, Eyer MK, and Backes WL (2013) Relationship between CYP1A2 localization and lipid microdomain formation as a function of lipid composition. Drug Metab Dispos 41:1896-1905.

Cawley GF, Batie CJ, and Backes WL (1995) Substrate-dependent competition of different P450 isozymes for limiting NADPH-cytochrome P450 reductase. Biochemistry 34:1244-1247.

Cawley GF, Zhang S, Kelley RW, and Backes WL (2001) Evidence supporting the interaction of CYP2B4 and CYP1A2 in microsomal preparations. Drug Metab Dispos 29:1529-1534.

Dacres H, Wang J, Dumancic MM, and Trowell SC (2010) Experimental determination of the Förster distance for two commonly used bioluminescent resonance energy transfer pairs. Anal Chem 82:432-435.

Davydov DR (2011) Microsomal monooxygenase as a multienzyme system: the role of P450-P450 interactions. Expert Opin Drug Metab Toxicol 7:543-558.

Davydov DR, Davydova NY, Sineva EV, and Halpert JR (2015) Interactions among cytochromes P450 in microsomal membranes: oligomerization of cytochromes P450 3A4, 3A5, and 2E1 and its functional consequences. J Biol Chem 290:3850-3864.

Davydov DR, Davydova NY, Sineva EV, Kufareva I, and Halpert JR (2013) Pivotal role of P450P450 interactions in CYP3A4 allostery: the case of $\alpha$-naphthoflavone. Biochem J 453:219-230.

Davydov DR, Petushkova NA, Bobrovnikova EV, Knyushko TV, and Dansette P (2001) Association of cytochromes P450 1A2 and 2B4: are the interactions between different P450 species involved in the control of the monooxygenase activity and coupling? Adv Exp Med Biol 500: 335-338.

Dutton DR, McMillen SK, Sonderfan AJ, Thomas PE, and Parkinson A (1987) Studies on the ratedetermining factor in testosterone hydroxylation by rat liver microsomal cytochrome P-450: evidence against cytochrome P-450 isozyme:isozyme interactions. Arch Biochem Biophys 255: 316-328.

Fernando H, Halpert JR, and Davydov DR (2008) Kinetics of electron transfer in the complex of cytochrome P450 3A4 with the flavin domain of cytochrome P450BM-3 as evidence of functional heterogeneity of the heme protein. Arch Biochem Biophys 471:20-31.

Guengerich FP (2006) A malleable catalyst dominates the metabolism of drugs. Proc Natl Acad Sci USA 103:13565-13566.

Hazai E and Kupfer D (2005) Interactions between CYP2C9 and CYP2C19 in reconstituted binary systems influence their catalytic activity: possible rationale for the inability of CYP2C19 to catalyze methoxychlor demethylation in human liver microsomes. Drug Metab Dispos 33: $157-164$.

Hu G, Johnson EF, and Kemper B (2010) CYP2C8 exists as a dimer in natural membranes. Drug Metab Dispos 38:1976-1983.

Jamakhandi AP, Kuzmic P, Sanders DE, and Miller GP (2007) Global analysis of protein-protein interactions reveals multiple CYP2E1-reductase complexes. Biochemistry 46:10192-10201.

James JR, Oliveira MI, Carmo AM, Iaboni A, and Davis SJ (2006) A rigorous experimental framework for detecting protein oligomerization using bioluminescence resonance energy transfer. Nat Methods 3:1001-1006.

Kaminsky LS and Guengerich FP (1985) Cytochrome P-450 isozyme/isozyme functional interactions and NADPH-cytochrome P-450 reductase concentrations as factors in microsomal metabolism of warfarin. Eur J Biochem 149:479-489.

Kelley RW, Cheng D, and Backes WL (2006) Heteromeric complex formation between CYP2E1 and CYP1A2: evidence for the involvement of electrostatic interactions. Biochemistry 45: $15807-15816$.

Kelley RW, Reed JR, and Backes WL (2005) Effects of ionic strength on the functional interactions between CYP2B4 and CYP1A2. Biochemistry 44:2632-2641.

Lynch T and Price A (2007) The effect of cytochrome P450 metabolism on drug response, interactions, and adverse effects. Am Fam Physician 76:391-396.

Marohnic CC, Huber WJ, III, Connick JP, Reed JR, McCammon K, Panda SP, Martásek P, Backes WL, and Masters BSS (2011) Mutations of human cytochrome P450 reductase differentially modulate heme oxygenase-1 activity and oligomerization. Arch Biochem Biophys 513:42-50.

Mercier JF, Salahpour A, Angers S, Breit A, and Bouvier M (2002) Quantitative assessment of $\beta_{1^{-}}$and $\beta_{2}$-adrenergic receptor homo- and heterodimerization by bioluminescence resonance energy transfer. J Biol Chem 277:44925-44931.

Miwa GT, West SB, Huang MT, and Lu AYH (1979) Studies on the association of cytochrome $\mathrm{P}-450$ and NADPH-cytochrome $\mathrm{c}$ reductase during catalysis in a reconstituted hydroxylating system. J Biol Chem 254:5695-5700.

Park JW, Reed JR, and Backes WL (2015) The localization of cytochrome P450s CYP1A1 and CYP1A2 into different lipid microdomains is governed by their $\mathrm{N}$-terminal and internal protein regions. J Biol Chem 290:29449-29460.

Park JW, Reed JR, Brignac-Huber LM, and Backes WL (2014) Cytochrome P450 system proteins reside in different regions of the endoplasmic reticulum. Biochem J 464:241-249. 
Peterson JA, Ebel RE, O'Keeffe DH, Matsubara T, and Estabrook RW (1976) Temperature dependence of cytochrome P-450 reduction. A model for NADPH-cytochrome P-450 reductase: cytochrome P-450 interaction. J Biol Chem 251:4010-4016.

Reed JR and Backes WL (2017) Physical studies of P450-P450 interactions: predicting quaternary structures of P450 complexes in membranes from their X-ray crystal structures. Front Pharmacol 8:28.

Reed JR, Cawley GF, and Backes WL (2011) Inhibition of cytochrome P450 1A2-mediated metabolism and production of reactive oxygen species by heme oxygenase-1 in rat liver microsomes. Drug Metab Lett 5:6-16.

Reed JR, Connick JP, Cheng D, Cawley GF, and Backes WL (2012) Effect of homomeric P450-P450 complexes on P450 function. Biochem J 446:489-497.

Reed JR, Eyer M, and Backes WL (2010) Functional interactions between cytochromes P450 1A2 and $2 \mathrm{~B} 4$ require both enzymes to reside in the same phospholipid vesicle: evidence for physical complex formation. J Biol Chem 285:8942-8952.

Sansen S, Yano JK, Reynald RL, Schoch GA, Griffin KJ, Stout CD, and Johnson EF (2007) Adaptations for the oxidation of polycyclic aromatic hydrocarbons exhibited by the structure of human P450 1A2. J Biol Chem 282:14348-14355.

Subramanian M, Low M, Locuson CW, and Tracy TS (2009) CYP2D6-CYP2C9 protein-protein interactions and isoform-selective effects on substrate binding and catalysis. Drug Metab Dispo 37:1682-1689.
Subramanian M, Tam H, Zheng H, and Tracy TS (2010) CYP2C9-CYP3A4 protein-protein interactions: role of the hydrophobic N terminus. Drug Metab Dispos 38:1003-1009.

West SB and Lu AYH (1972) Reconstituted liver microsomal enzyme system that hydroxylates drugs, other foreign compounds and endogenous substrates. V. Competition between cytochromes P-450 and P-448 for reductase in 3,4-benzpyrene hydroxylation. Arch Biochem Biophys 153:298-303.

Yamada M, Ohta Y, Bachmanova GI, Nishimoto Y, Archakov AI, and Kawato S (1995) Dynamic interactions of rabbit liver cytochromes P450IA2 and P450IIB4 with cytochrome $b_{5}$ and NADPH-cytochrome P450 reductase in proteoliposomes. Biochemistry 34:10113-10119.

Yasukochi Y and Masters BS (1976) Some properties of a detergent-solubilized NADPHcytochrome $c$ (cytochrome P-450) reductase purified by biospecific affinity chromatography. $J$ Biol Chem 251:5337-5344.

Zanger UM and Schwab M (2013) Cytochrome P450 enzymes in drug metabolism: regulation of gene expression, enzyme activities, and impact of genetic variation. Pharmacol Ther 138:103-141.

Address correspondence to: Wayne L. Backes, Department of Pharmacology and Experimental Therapeutics, Louisiana State University Health Sciences Center, 533 Bolivar Street, New Orleans, LA 70112. E-mail: wbacke@lsuhsc.edu 